\title{
Lungenkrebs-Screening deckt frühe Krankheitsstadien auf
}

Die 2010 publizierten Daten des National Lung Screening Trials hatten eine 20\%-ige Reduktion der Mortalität bei einem Kollektiv mit mehr als 50000 Personen durch 3 Low-DoseThorax-Computertomografien in jährlichen Abständen im Vergleich zu konventionellen Summationsradiografien gezeigt. Z. Saghir et al. haben nun ein jährliches CT-Screening mit dem konventionellen Vorgehen über 5 Jahre verglichen.

Thorax 2012; 67: 296-301

Die Population der Studie bestand aus 2104 Rauchern und Ex-Rauchern mit einem Alter von 50-70 Jahren, mindestens 20 Packungsjahren und gutem körperlichen Zustand, bei denen im vergangenen Jahr keine Thorax-CT durchgeführt wurde. Die Teilnehmer wurden für jährliche LowDose-Thorax-CT-Untersuchungen oder die übliche symptomorientierte Behandlung durch Haus- und Fachärzte (Kontrollgruppe) im Verhältnis 1:1 randomisiert. Alle unterzogen sich einer jährlichen klini- schen Untersuchung mit Anamnese, körperlichem Status und Lungenfunktion. Probanden der Interventionsgruppe mit radiologischen Knoten zwischen 5 und $15 \mathrm{~mm}$ erhielten nach 3 Monaten einen Kontrollscan, wenn keine Benignitätskriterien vorlagen. Waren die Knoten über 15 mm groß, wurden diese diagnostisch abgeklärt.

Innerhalb der 5-jährigen Nachbeobachtungszeit fanden sich in der Interventions- gruppe deutlich mehr Lungenkrebsfälle (69 vs. 24; p < 0,001), davon 48 vs. $21 \mathrm{im}$ Limited-Disease-Stadium. Die Zahl der fortgeschrittenen Lungenkarzinome unterschied sich mit 21 vs. 16 nicht signifikant. Während der Nachbeobachtungszeit starben insgesamt 61 vs. 42 Teilnehmer $(p=0,059)$. Davon war bei 15 vs. 11 Personen Lungenkrebs die Todesursache $(p=0,428)$. Die Autoren erklären die fehlende Signifikanz mit der geringeren Probengröße und der etwas kürzeren Nachbeobachtungszeit. Sie geben jedoch auch zu bedenken, dass sich diese - bedingt durch die kurze durchschnittliche Überlebensdauer bei fortgeschrittenem Lungenkarzinom von 13-17 Monaten - bereits innerhalb der 5-jährigen Studiendauer hätte zeigen müssen.

\section{Fazit}

Ein Low-Dose-CT-Screening des Thorax decke mehr Frühstadien des Lungenkarzinoms auf, jedoch nicht mehr fortgeschrittene Erkrankungsfälle, so die Autoren. Ebenfalls werde die Mortalität nicht reduziert. Dies könne z. B. auf eine Überdiagnose nicht therapiebedürftiger Fälle hinweisen.

\section{Dr. Peter Pommer, Oberammergau}

atopischen Ekzem (7\%), in der Kontrollgruppe waren es 39 (12\%; adjustiertes relatives Risiko [RR] 0,64; 95\%-Konfidenzintervall [KI] 0,40-1,02; $\mathrm{p}=0,06$ ). Darüber hinaus hatten weniger Kinder in der Interventionsgruppe eine Sensibilisierung gegenüber Hühnereiern (9 vs. $15 \%$; adjustiertes RR 0,62; 95\%-KI 0,41$1,93 ; \mathrm{p}=0,02)$, auch wenn der Unterschied bei Nahrungsmittelallergien insgesamt nicht relevant war.

\section{Fazit}

Eine Supplementation mit Omega3-Fettsäuren während der Schwangerschaft kann bei Kindern im 1. Lebensjahr die Häufigkeit von Allergien insgesamt zwar nicht messbar senken, die Verminderung von atopischen Ekzemen scheint aber bedeutsam, so die Autoren. Weitere allergische Erkrankungen zeigen ein Häufigkeitsmaximum erst bis zum 15. Lebensjahr, sodass die Effekte des Fischöls hier später zu erwarten seien. Eine erneute Untersuchung der Kinder am Ende ihres 3. und 6. Lebensjahres ist geplant.

Dr. Elke Ruchalla, Trossingen 